[10] M. Reidel and J. Rajski, "Fault coverage analysis of RAM test algorithms," in Proc. VLSI Test Symp., 1995, pp. 227-234.

[11] A. Jee and F. Ferguson, "Carafe: An inductive fault analysis tool for CMOS VLSI circuits," in Proc. VLSI Test Symp., 1993, pp. 92-98.

[12] V. Kim, "Manufacturing test simulator for chips," Ph.D. dissertation, Dept. Elect. Eng., Colorado State Univ., Fort Collins, 1997.

[13] R. Dekker, "Fault modeling and self-test of static random access memories," Delft University of Technology, Delft, The Netherlands, Tech. Rep. TUD report 1-68340-28(1987)25, 1987.

\section{Global Routing with Crosstalk Constraints}

\author{
Hai Zhou and D. F. Wong
}

\begin{abstract}
Due to the scaling down of device geometry and increasing of frequency in deep submicron designs, crosstalk between interconnection wires has become an important issue in very large scale integration (VLSI) layout design. In this paper, we consider crosstalk avoidance during global routing. We present a global routing algorithm based on a new Steiner tree formulation and the Lagrangian relaxation technique. We also give theoretical results on the complexity of the problem.
\end{abstract}

Index Terms-Crosstalk, integrated circuit interconnections, layout, routing.

\section{INTRODUCTION}

With very large scale integration (VLSI) fabrication entering the deep submicron era, devices and interconnection wires are being placed at an ever increasing proximity. Reduction in the interconnect and transistor switching delays results in faster signal transition times. All these factors increase the coupling effect (inductive and capacitive) between interconnection wires. Increased coupling effect not only increases signal delays, but also decreases signal integrity due to transmission line behavior. This phenomenon is called crosstalk [1].

Most previous work on crosstalk avoidance is focused on detailed routing [2]-[7], where the estimation of crosstalk is accurate but the flexibility to avoid it is restricted. Therefore, it is often not possible to find a crosstalk-feasible solution in detailed routing if global routing is crosstalk-blinded.

In order to make a global routing solution crosstalk risk-free, Xue et al. [8] proposed a post global routing adjustment procedure to modify a given routing solution. It used a gridded model and assumed that crosstalk only exists between adjacent wires. That is, if there is a spare track between two wires, then there is no crosstalk between them. The crosstalk risk estimation is divided into two coupled procedures: bound partitioning and net ordering. Bound partitioning partitions the crosstalk bound of each net into the regions it routes through. Net ordering orders the nets in each region to use as few spare tracks as possible. Their approach is region-based, that is, crosstalk risks are defined by the regions requiring extra tracks and

Manuscript received December 3, 1998; revised July 26, 1999 This paper was recommended by Associate Editor C.-K. Cheng.

H. Zhou is with the Advanced Technology Group, Synopsys, Inc., Mountain View, CA 94043 USA

D. F. Wong is with the Department of Computer Sciences, University of Texas, Austin, TX 78712-1188 USA.

Publisher Item Identifier S 0278-0070(99)09473-7.

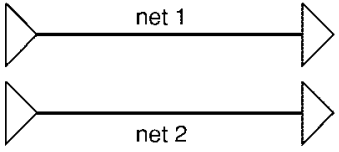

(a)

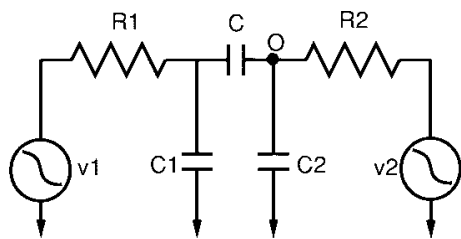

(b)
Fig. 1. Modeling two coupling nets.

the number of extra tracks needed. If a region still requires extra tracks after bound partitioning and net ordering, some nets in the region are ripped up and rerouted through other regions.

In this paper, we consider crosstalk avoidance during global routing. We use a crosstalk model which is more general than that of [8]. In order to have an accurate crosstalk estimation, we extend global routing to include layer and track assignments. Based on this extension, a novel Crosstalk-Constrained Global Routing problem is defined. Many subproblems of this problem are shown to be NP-hard. Therefore, a two-stage heuristic approach is used to control crosstalk. First, an initial solution is constructed based on a new Steiner tree formulation to minimize the total crosstalk. After that, crosstalk on each net is estimated. The nets having crosstalk violations are then ripped up. They are rerouted by a Lagrangian relaxation technique to satisfy crosstalk constraints.

The rest of the paper is organized as follows. Section II introduces the general crosstalk model we use. Section III extends the global routing to include layer/track assignment and defines the crosstalkconstrained global routing problem. In Section IV we show that many subproblems of the crosstalk-constrained global routing problem are NP-hard. Section V presents our global router for crosstalk control. Finally, Section VI concludes the paper with experimental results.

\section{Crosstalk Model}

Consider two coupling nets as shown in Fig. 1(a). We can model them by a circuit shown in Fig. 1(b). In this figure, $v_{1}, v_{2}$ represent the input waveforms of source drivers, $R_{1}, R_{2}$ represent the driver resistances, $C$ represents the coupling capacitance between the nets, and $C_{1}, C_{2}$ represent all other capacitances besides $C$. The crosstalk effect on net 2 can be thought of as the difference of voltages on node $O$ with or without the signal switching in net 1 . Here, we can see that besides coupling capacitance $C$, crosstalk effect also depends on driver strengths $\left(R_{1}\right.$ and $\left.R_{2}\right)$, other load capacitances $\left(C_{1}\right.$ and $\left.C_{2}\right)$, and the input waveforms $\left(v_{1}\right.$ and $\left.v_{2}\right)$.

Among all parameters affecting crosstalk effect, we assume that only the coupling capacitance is controllable by layout design. Other parameters are either not changeable or not preferred to be changed. However, they are useful information for crosstalk control in layout design. For example, if we know net 1 and net 2 will never switch at the same time, then we can safely route them together since the crosstalk effect will be zero even they are heavily coupled. We model all parameters except coupling capacitance by crosstalk coefficient. For each net $i$, the crosstalk coefficient from net $j$ is a real number $e_{i j} \in[0,1]$ which is the crosstalk on net $i$ contributed by one unit of coupling capacitance from net $j$. For example, if $e_{i j}=2 e_{i k}$, then the crosstalk induced on net $i$ by net $j$ is twice of that by net $k$ if they have the same coupling capacitance.

For one net, crosstalk effects from other nets may not happen at the same time. But characterizing all cases requires exhaustive timing 


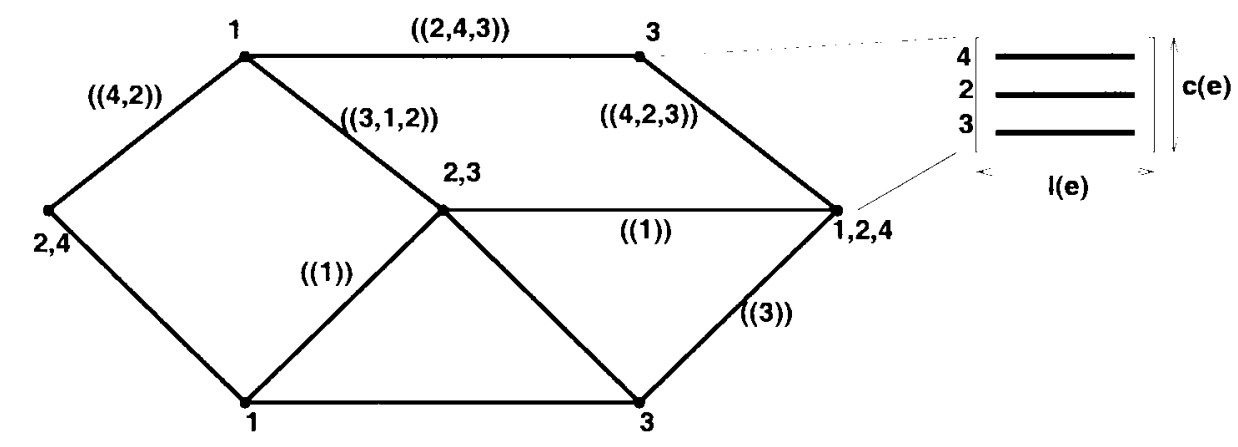

Fig. 2. An extended global routing solution.

analysis, which in turn depends on crosstalk. Therefore, in the worst case, we can use the summation of all effects from other nets as the total crosstalk on one net. That is

$$
X_{i}=\sum_{j \neq i} e_{i j} C_{i j}
$$

where $C_{i j}$ is the coupling capacitance between net $i$ and net $j$.

Generally speaking, each element in a chip is coupled with every other element. But coupling capacitance decreases rapidly when an element is out of the neighborhood of the other element. For interconnect, coupling capacitance between perpendicular wires is also very small. Therefore, we assume coupling capacitance only exists between neighboring parallel wires and is given by the following formula:

$$
C=\alpha \frac{\text { length }}{\text { distance }^{\beta}}
$$

where $\beta$ is a constant which was estimated to be about 2 in [9].

\section{PRoblem Formulation}

In traditional design flow, routing is divided into global and detailed routing. In global routing, a set of regions for each net to go through is decided. In detailed routing, actual routes in each region is then computed. From the previous section, we know coupling is critically dependent on wire adjacency. However, in traditional global routing, for each net, only routing regions it going through are decided. The adjacency information in each region is not available. Therefore, it is not possible to get accurate crosstalk estimation in that situation.

In order to estimate crosstalk during global routing, some "detailed" information must be known. However, doing the whole task of global and detailed routing together would be very complicated. Therefore, the idea is to do a "simplified" detailed routing "on the fly" during the process of global routing. This detailed routing must be simple enough to be merged into global routing process, and at the same time, must be detailed enough for crosstalk estimation.

In global routing, the routing area is usually divided into an array of rectangular regions. A vertex is placed at the center of each region and an edge is used to connect the vertices of neighboring regions. Thus, an edge also represents a rectangular area between the center lines of two neighboring regions. Actually, in multilayer designs, this area may consist of more than one layer. We assume that each layer is composed of tracks with equal spacing and each track can be used only by one net. During global routing, besides deciding which regions a net goes through, layer and track assignments in each region are also decided. Formally, we have an undirected graph $G=(V, E)$, where each edge $e$ represents a region $r(e)$ which has a length $l(e)$ and may have $k$ layers each with a capacity $c_{i}(e)$, for $1 \leq i \leq k$. Also specified are $n$ nets each of which is a subset of
$V$. An extended global routing solution is an edge labeling $S$ of the form $S(e)=\left(s_{1}, s_{2}, \ldots, s_{k}\right)$, where each $s_{i}$ for $1 \leq i \leq k$ is a sequence of nets. For $1 \leq i \leq n$, the edges including $i$ must form a Steiner tree for net $i$. If $s_{i}=(\ldots, 2,1,3, \ldots)$, this means that nets 1,2 , and 3 are routed through region $r(e)$ and placed in layer $i$ in the order 2, 1, 3. For example, an extended global routing solution is shown in Fig. 2, where each region has only one layer.

Given an extended global routing solution, the adjacency information is known in each edge. Suppose nets $i$ and $j$ are adjacent on layer $l$ of edge $e$, the coupling capacitance between the parallel wires can be computed as $C=\alpha\left(l(e) /\left(c_{l}(e) / N_{l}(e)\right)^{\beta}\right)$, where $N_{l}(e)$ represents the number of nets on layer $l$ of edge $e$. In this way, crosstalk on each net can be computed. Using $X_{i}(S)$ to represent crosstalk on net $i$ under an extended global routing solution $S$, the problem we need to solve can be defined as follows.

Problem 1-Crosstalk-Constrained Global Routing (CCGR): Given a routing graph $G=(V, E), n$ nets, and their crosstalk constraints $C_{1}, \ldots, C_{n}$, find an extended global routing solution $S$ such that $X_{i}(S) \leq C_{i}$ for all $1 \leq i \leq n$.

\section{COMPLEXIty Results}

In the CCGR problem, both routing topology and adjacency relations in each region can be changed. Therefore, the following methods can be use to reduce crosstalk: shielding (inserting a spare wire between coupling nets), track assignment (changing neighboring relations of nets) and layer assignment (distributing coupling wires to different layers). Restricting the CCGR problem to each of these methods, we can formulate the following subproblems.

Problem 2-Shielding for Crosstalk Control: Suppose the routing topology and layer/track assignments in all regions are fixed, and only one shielding wire can be inserted in each region. Find a shielding position in each region such that each net satisfies its crosstalk constraint.

Problem 3-Track Assignment for Crosstalk Minimization: Suppose the routing topology is fixed, and so are track assignments in all regions except one. Find a track assignment in that region such that the total crosstalk on all nets is minimized.

Problem 4-Track Assignment for Crosstalk Control: Suppose the routing topology is fixed, and so are track assignments in all regions except one. Find a track assignment in that region such that each net satisfies its crosstalk constraint.

Problem 5-Layer Assignment for Crosstalk Control: Suppose there are only two layers in each region, and the routing topology and net orderings in all regions are fixed. Find a layer assignment in each region such that each net satisfies its crosstalk constraint.

As stated by the following theorem, all the above problems are intractable. 
Theorem 1: Problems 2-5 are all NP-hard.

Proof: We show NP-hardness of these four problems by reductions from two problems: PARTITION and HAMILTONIAN PATH. These problems are defined as follows [10].

\section{PARTITION}

INSTANCE: Finite set $A$ and a size $s(a) \in Z^{+}$for each $a \in A$.

QUESTION: Is there a subset $A^{\prime} \in A$ such that $\Sigma_{a \in A^{\prime}} s(a)=$ $\Sigma_{a \in A-A^{\prime}} s(a)$ ?

\section{HAMILTONIAN PATH}

INSTANCE: Graph $G=(V, E)$.

QUESTION: Does $G$ contain a Hamiltonian path?

Specifically, we reduce PARTITION to the problem of shielding for crosstalk control and the problem of layer assignment for crosstalk control, and reduce HAMILTONIAN PATH to the problem of track assignment for crosstalk minimization and the problem of track assignment for crosstalk control.

Let the problem of shielding for crosstalk control has three nets, $1-3$, which are routed by the same topology and layer assignment and fixed in the sequence 1, 2, 3 in each region. Also each region is one-to-one correspondent to an $a \in A$, that is, crosstalk between two adjacent wires in the region is $s(a)$. The crosstalk constraints are $C_{1}=C_{3}=\Sigma_{a \in A} s(s) / 2$, and $C_{2}=\infty$. It is easy to check that there is a solution to this problem of shielding for crosstalk control if and only if PARTITION has a yes answer.

Very similarly, let the problem of layer assignment for crosstalk control has three nets, 1-3, which are routed by the same topology and net ordering. The crosstalk between adjacent wires in a region is also $s(a)$ for each $a \in A$. The crosstalk constraints are the same as in the above reduction. It is also easy to check that there is a solution to this problem of layer assignment for crosstalk control if and only if PARTITION has a yes answer.

Given $G=(V, E)$ as an instance of HAMILTONIAN PATH, for each $v \in V$, we will have a net $v$ in the problem of track assignment for crosstalk minimization. For any pair of nets $u$ and $v$, its crosstalk coefficient $e_{u v}=e_{v u}=0$ if $(u, v) \in E$; otherwise $e_{u v}=e_{v u}=$ 1. Each of these nets is routed through different regions except in one region where all nets are routed together. It is easy to check that there is a track assignment with zero total crosstalk if and only if $G$ contains a Hamiltonian path.

We construct the problem of track assignment for crosstalk control exactly the same as in the above reduction. Furthermore, we set the crosstalk constraint for each net to be zero. In this case, it is also easy to check that there is a solution to the problem if and only if $G$ contains a Hamiltonian path.

\section{A Global Router with Crosstalk Control}

As shown in the previous section, many subproblems of the CCGR problem are NP-hard. Therefore, we use a heuristic to solve the problem. It is a sequential approach, that is, routing is done net by net. This approach consists of two stages. The first stage constructs a routing solution to minimizes the summation of crosstalk on all nets. Each time, a Steiner tree is computed for a net which minimizes the total crosstalk and its topology is then fixed for the remaining nets. In the second stage, crosstalk on each net in the initial solution is estimated. The nets having constraint violations are then ripped up. During rerouting, a method based on Lagrangian relaxation is used to balance crosstalk of each net according to its constraint. Since there is no forbidden region in the process, a new route can have the same topology as the old one but with different layer/track assignment. This means layer/track reassignment is also included in this procedure.

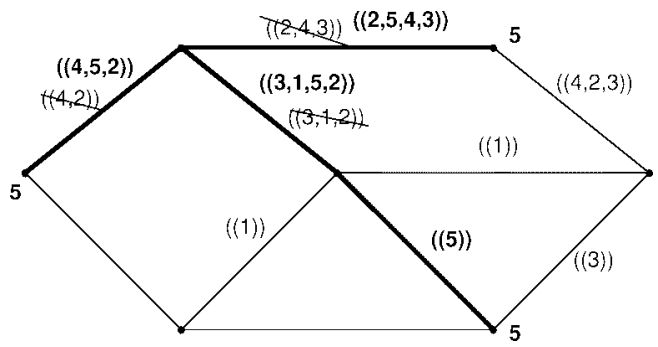

Fig. 3. Routing net 5.

\section{A. Minimum Crosstalk Steiner Tree}

Since a sequential routing approach is used, the kernel procedure is how to construct a Steiner tree and decide the layer/track assignment in each region such that the total crosstalk is minimize. Formally, this can be defined as the following problem.

Problem 6-Minimum Crosstalk Steiner Tree: Given a routing graph $G=(V, E)$ and an extended global routing solution $S$ for nets $1, \ldots, m-1$ which minimizes the total crosstalk, find an extended global routing solution $S^{\prime}$ for nets $1, \ldots, m$ such that routing topologies for nets $1, \ldots, m-1$ are kept and $X\left(S^{\prime}\right)=\Sigma_{i=1}^{m} X_{i}\left(S^{\prime}\right)$ is minimized.

Let $X^{e}(S)$ denote the summation of crosstalk on all wires in edge $e$ under solution $S$. The objective function in the above problem can be rewritten as follows:

$$
\begin{aligned}
X\left(S^{\prime}\right) & =\sum_{e \in E} X^{e}\left(S^{\prime}\right) \\
& =\sum_{e \in T_{m}} X^{e}\left(S^{\prime}\right)+\sum_{e \notin T_{m}} X^{e}\left(S^{\prime}\right) \\
& =\sum_{e \in T_{m}}\left(X^{e}\left(S^{\prime}\right)-X^{e}(S)\right)+\sum_{e \in E} X^{e}(S)
\end{aligned}
$$

where $T_{m}$ represents the Steiner tree for net $m$. For each edge $e$, $X^{e}\left(S^{\prime}\right)-X^{e}(S)$ is the difference of the minimum total crosstalk in edge $e$ before and after routing net $m$ through that edge. Given a set of nets going through an edge, the minimum crosstalk in the edge can be computed by an optimal layer/track assignment. Therefore, we can compute $X^{e}\left(S^{\prime}\right)-X^{e}(S)$ and use it as the cost of edge $e$. Since $\Sigma_{e \in E} X^{e}(S)$ in (3) is a constant, the minimum crosstalk Steiner tree problem becomes a minimum Steiner tree problem with the given edge costs.

Unfortunately, according to Theorem 1, layer/track assignment for crosstalk minimization in one region is NP-hard. Therefore, a heuristic is designed to do the job. It works as follows. First, we decide an ordering on all nets. Then, each net is added sequentially to the region according to the order. When adding one net, the existing nets keep their relative ordering, and the new net is inserted to a position which gives the minimum increase of crosstalk.

If we use the same net ordering for routing in the above heuristic, the layer/track assignments can be merged into the routing process. In this case, when computing edge costs, there is no need to do layer/track reassignments for the existing nets. Suppose $S$ is the given solution for nets $1, \ldots, m-1$, the cost of routing net $m$ through edge $e$ is the minimum increase of crosstalk by inserting net $m$ in one sequence of $S(e)$. A minimum Steiner tree based on these edge costs, together with the corresponding insertions, forms the new solution $S^{\prime}$. An example of the procedure is given in Fig. 3 .

In practice, crosstalk is not the only objective we want to minimize. We also need to consider wire length and congestion. In such case, we can use the following edge cost function:

$$
\operatorname{cost}(e)=\alpha \cdot \text { length }(e)+\beta \cdot \text { overflow }(e)^{2}+\gamma \cdot \operatorname{xtalk}(e)
$$




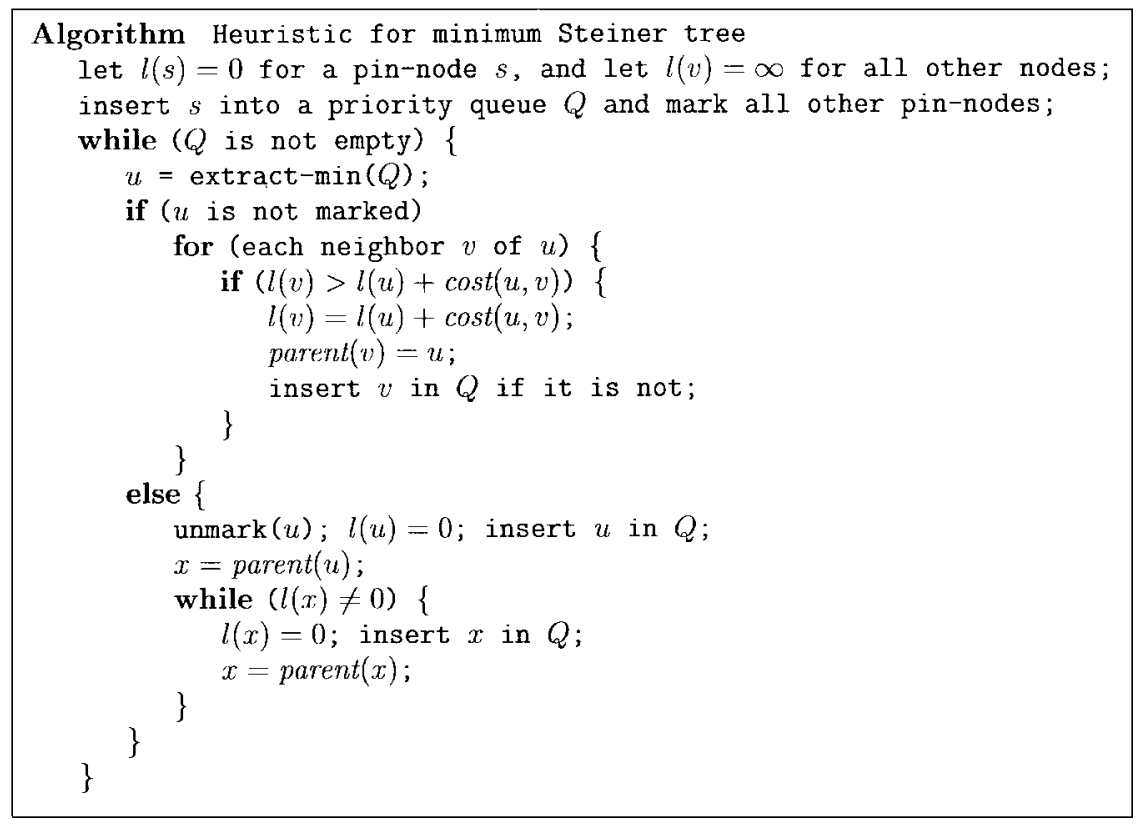

Fig. 4. Minimum Steiner tree heuristic.

where $\operatorname{overflow}(e)$ is the needed extra space exceeding the capacity of edge $e$, and $x$ talk $(e)$ is the increase of crosstalk on edge $e$. Based on this cost function, any Steiner tree heuristic on general weighted graph can be used to construct a Steiner tree which simultaneously optimizes the wire length, congestion, and crosstalk.

The Steiner tree heuristic we use in our global router is based on the shortest path and the minimum spanning tree. According to [11], these heuristics are guaranteed to have solutions within $2(1-1 / l)$ of the optimum, where $l$ is the number of leaves in the optimal solution. We use Dijkstra's algorithm [12] for the shortest path and Prim's algorithm [12] for the minimum spanning tree. The reason we use Prim's algorithm instead of Kruskal's is that Dijkstra's algorithm and Prim's algorithm are both working in a tree expansion fashion, hence, can be easily combined. The pseudocode of the procedure is given in Fig. 4.

\section{B. Rip-up and Reroute Based on Lagrangian Relaxation}

Given an initial solution constructed by the minimum crosstalk Steiner tree algorithm, nets with crosstalk violations are ripped up. We reroute them one net at a time in the order of decreasing violations. When routing a net, besides crosstalk on the current net, extra crosstalk can be induced on previously routed nets. Therefore, we need to solve the following problem.

Problem 7-Rerouting for Crosstalk Control: Given a routing graph $G=(V, E)$ and an extended global routing solution $S$ for nets $1, \ldots, m-1$ such that $X_{i}(S) \leq C_{i}$ for $1 \leq i \leq m-1$, find an extended global routing solution $S^{\prime}$ for nets $1, \ldots, m$ such that routing topologies of nets $1, \ldots, m-1$ are kept, the wire length is minimized, and $X_{i}\left(S^{\prime}\right) \leq C_{i}$ for all $1 \leq i \leq m$.

Compared with the minimum crosstalk Steiner tree problem which minimizes the total crosstalk, the above problem requires to balance the crosstalk on each net according to its constraint, hence is more complicated. However, we can use Lagrangian relaxation technique to satisfy all constraints simultaneously. By relaxing constraints into objective function, we have the following problem.

Problem 8-Lagrangian Subproblem (LSP): Given a routing graph $G=(V, E)$, an extended global routing solution $S$ for nets $1, \ldots, m-1$, and constants $\mu_{1}, \ldots, \mu_{m}$, find an extended global routing solution $S^{\prime}$ for nets $1, \ldots, m$ such that the routing topology for nets $1, \ldots, m-1$ is kept, and length $\left(S^{\prime}\right)+\Sigma_{i=1}^{m} \mu_{i}\left(X_{i}\left(S^{\prime}\right)-C_{i}\right)$ is minimized, where length $\left(S^{\prime}\right)$ is the wire length in solution $S^{\prime}$.

Let length $h_{i}\left(S^{\prime}\right)$ be the wire length of net $i$ under solution $S^{\prime}$. The objective function of above problem can be rewritten as follows:

$$
\begin{aligned}
\text { length }\left(S^{\prime}\right)+\sum_{i=1}^{m} \mu_{i} & \left(X_{i}\left(S^{\prime}\right)-C_{i}\right) \\
& =\sum_{i=1}^{m}\left(\text { length }_{i}\left(S^{\prime}\right)+\mu_{i} X_{i}\left(S^{\prime}\right)\right)-\sum_{i=1}^{m} \mu_{i} C_{i}
\end{aligned}
$$

Since $\Sigma_{i=1}^{m} \mu_{i} C_{i}$ is a constant, by treating length $h_{i}\left(S^{\prime}\right)+\mu_{i} X_{i}\left(S^{\prime}\right)$ as "crosstalk" on net $i$, the Lagrangian subproblem becomes the minimum crosstalk Steiner tree problem, hence can be solved by the same algorithm.

Let $L\left(\mu_{1}, \ldots, \mu_{m}\right)$ denote the optimal solution of the Lagrangian subproblem. For any $\mu_{i} \geq 0,1 \leq i \leq m$, we have

$$
\begin{aligned}
& L\left(\mu_{1}, \ldots, \mu_{m}\right) \\
& =\min _{S^{\prime}}\left\{\text { length }\left(S^{\prime}\right)+\sum_{i=1}^{m} \mu_{i}\left(X_{i}\left(S^{\prime}\right)-C_{i}\right)\right\} \\
& \leq \min _{S^{\prime}}\left\{\text { length }\left(S^{\prime}\right)+\sum_{i=1}^{m} \mu_{i}\left(X_{i}\left(S^{\prime}\right)-C_{i}\right): X_{i}\left(S^{\prime}\right)\right. \\
& \left.\leq C_{i}, 1 \leq i \leq m\right\} \\
& \leq \min _{S^{\prime}}\left\{\text { length }\left(S^{\prime}\right): X_{i}\left(S^{\prime}\right) \leq C_{i}, 1 \leq i \leq m\right\} .
\end{aligned}
$$

This means that, for any fixed $\mu_{i} \geq 0,1 \leq i \leq m$, the solution of the Lagrangian subproblem $L\left(\mu_{1}, \ldots, \mu_{m}\right)$ is a lower bound of the rerouting for crosstalk control problem. Therefore, if we find $\mu_{1}^{*}, \ldots, \mu_{m}^{*}$ such that

$$
L\left(\mu_{1}^{*}, \ldots, \mu_{m}^{*}\right)=\min _{S^{\prime}}\left\{\text { length }\left(S^{\prime}\right): X_{i}\left(S^{\prime}\right) \leq C_{i}, 1 \leq i \leq m\right\}
$$

we know that $S^{\prime}$ is an optimal solution. Therefore, we need to solve the following problem. 
TABLE I

EXPERIMENTAL RESULTS

\begin{tabular}{l|r|r|r|r|r|r|r|r}
\hline \multirow{2}{*}{$\begin{array}{c}\text { test } \\
\text { problems }\end{array}$} & \multicolumn{2}{|c|}{$\begin{array}{c}\text { Traditional } \\
\text { GR }\end{array}$} & \multicolumn{2}{c}{$\begin{array}{c}\text { post-GR layer/track } \\
\text { assignment }\end{array}$} & \multicolumn{4}{c}{ our approach } \\
\cline { 2 - 9 } \#vio. & max. vio & \#vio. & Max. vio & \#vio. & Max. vio & \#vio. & Max. vio \\
\hline ami49.1.a & 329 & 285.29 & 301 & 191.91 & 121 & 81.46 & 1 & 0.84 \\
ami49.1.b & 252 & 86.27 & 143 & 35.46 & 46 & 15.23 & 1 & 0.14 \\
ami49.1.c & 303 & 114.50 & 265 & 46.78 & 146 & 25.93 & 3 & 0.90 \\
ami49.2 & 325 & 100.46 & 283 & 46.16 & 156 & 16.92 & 2 & 0.19 \\
ami33.1 & 70 & 82.15 & 65 & 31.90 & 35 & 8.97 & 5 & 0.39 \\
ami33.2 & 64 & 83.07 & 55 & 31.87 & 24 & 8.89 & 1 & 0.19 \\
apte.a & 19 & 21.93 & 11 & 7.56 & 3 & 4.54 & 0 & 0.00 \\
apte.b & 17 & 5.15 & 11 & 2.10 & 5 & 1.74 & 0 & 0.00 \\
xerox.1 & 49 & 5.16 & 9 & 4.34 & 3 & 3.83 & 2 & 0.08 \\
xerox.2 & 57 & 17.17 & 18 & 5.87 & 2 & 0.32 & 0 & 0.00 \\
xerox.3 & 70 & 49.70 & 34 & 31.23 & 22 & 15.98 & 0 & 0.00 \\
\hline
\end{tabular}

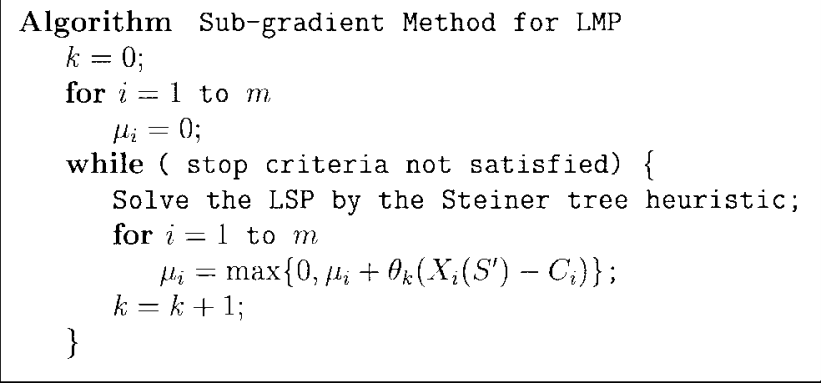

Fig. 5. Pseudocode of subgradient method.

Problem 9-Lagrangian Multiplier Problem (LMP):

$$
\begin{array}{ll}
\text { Maximize } & L\left(\mu_{1}, \ldots, \mu_{m}\right) \\
\text { subject to } & \mu_{i} \geq 0, \forall 1 \leq i \leq m
\end{array}
$$

The above problem has a good property for optimization: it is a convex program. Nonlinear programming techniques can be used to solve this problem. Since $L\left(\mu_{1}, \ldots, \mu_{m}\right)$ as a function is not everywhere differentiable, we will use the subgradient method to solve it. The pseudocode of the subgradient method is given in Fig. 5.

The general theory for subgradient method states that if $\theta_{k} \rightarrow 0$ and $\sum_{j=1}^{k} \theta_{j} \rightarrow \infty$ as $k \rightarrow \infty$, it will converge to an optimal solution [13]. Intuitively, the procedure can be explained as follows. First, we give a relative weight to each net as the importance of its crosstalk control. Then we minimize a weighted sum of crosstalk on all nets. After that, we estimate the crosstalk on each net. For those nets with violations, we increase their weights according to the violations; for those nets with slacks, we decrease their weights according to the slacks.

The only problem with this approach is that there may exist a "duality gap" between the problem of rerouting for crosstalk control and the corresponding Lagrangian multiplier problem. That is, it is possible that

$$
\min \left\{\text { length }\left(S^{\prime}\right): X_{i}\left(S^{\prime}\right) \leq C_{i}\right\}>\max L\left(\mu_{1}, \ldots, \mu_{m}\right)
$$

Furthermore, solving the problem of rerouting for crosstalk control is just one step in the whole process of rerouting. Hence, it is not necessary to spend too much time to get an optimal solution. Therefore, we set up an upper bound on the number of iterations. During the iterations, we also record the best solution, that is, the ever seen solution $S^{\prime}$ with shortest wire length such that $X_{i}\left(S^{\prime}\right) \leq C_{i}$ or a solution $S^{\prime}$ such that $\max _{i}\left(X_{i}\left(S^{\prime}\right)-C_{i}\right)$ is minimum. If the subgradient method does not give the optimal solution within those iterations, we use the recorded best solution.

\section{EXPERIMENTAL RESULTS}

We implement the global router described in Section V on a Solaris/PC platform. Five circuits from the Collaborative Benchmarking Lab at North Carolina State University (CBL/NCSU) building block benchmarks: ami33, ami49, apte, hp, and xerox are used. Since these benchmarks do not come with placement information, we use a floorplaner based on simulated annealing [14] to give placements. Using different parameters, we obtain two different placements for both ami33 and ami49: ami33.1, ami33.2, ami49.1, ami49.2, and three different placements for xerox: xerox.1, xerox.2, xerox.3. Using different minimum track spacing and crosstalk constraints, different test problems are produced for the same placement, which are indicated by the suffixes: $a, b$, etc.

In order to measure the effectiveness of our global router, we compare it with two other routing approaches. "Traditional GR" only considers wire length and overflow, and nets are ordered sequentially in each region. "Post-GR layer/track assignment" uses the same topologies as in traditional GR but use the greedy heuristic given in Section V-A to do layer/track assignment in each region. For each approach, the numbers of nets having crosstalk violations and the maximum violations are reported in Table I. For our global router, both results after the first stage (i.e., minimizing total crosstalk) and the second stage (i.e., rip-up and reroute by Lagrangian relaxation) are reported. As we can see, after rip-up and reroute, almost all violations are solved. For those few nets which still have violations, the violations are so small that it could be easily solved in detailed routing.

\section{REFERENCES}

[1] H. B. Bakoglu, Circuits, Interconnections, and Packaging for VLSI. Reading, MA: Addison-Wesley, 1990.

[2] K. Chaudhary, A. Onozawa, and E. S. Kuh, "A spacing algorithm for performance enhancement and cross-talk reduction," in Proc. Int. Conf. Computer-Aided Design, Santa Clara, CA, Nov. 1993, pp. 697702.

[3] T. Gao and C. L. Liu, "Minimum crosstalk channel routing," in Proc. Int. Conf. Computer-Aided Design, Santa Clara, CA, Nov. 1993, pp. 692-696.

[4] _ "Minimum crosstalk switchbox routing," in Proc. Int. Conf. Computer-Aided Design, San Jose, CA, Nov. 1994, pp. 610615. 
[5] D. A. Kirkpatrick and A. L. Sangiovanni-Vincentelli, "Techniques for crosstalk avoidance in the physical design of high-performance digital systems," in Proc. Int. Conf. Computer-Aided Design, San Jose, CA, Nov. 1994, pp. 616-619.

[6] S. Thakur, K.-Y. Chao, and D. F. Wong, "An optimal layer assignment algorithm for minimizing crosstalk for three layer VHV channel routing," in Proc. Int. Symp. Circuits and Systems, Apr. 1995, pp. I207-I-210.

[7] H. Zhou and D. F. Wong, "An optimal algorithm for river routing with crosstalk constraints," in Proc. Int. Conf. Computer-Aided Design, San Jose, CA, 1996, pp. 310-315.

[8] T. Xue, E. S. Kuh, and D. Wang, "Post global routing crosstalk risk estimation and reduction," in Proc. Int. Conf. Computer-Aided Design, 1996, pp. 302-309.
[9] T. Sakurai and K. Tamaru, "Simple formulas for two and three dimensional capacitance," IEEE Trans. Electron Devices, pp. 183-185, 1983.

[10] M. R. Garey and D. S. Johnson, Computers and Intractability. San Francisco, CA: Freeman, 1979.

[11] F. K. Hwang, D. S. Richards, and P. Winter, The Steiner Tree Problem. Amsterdam, The Netherlands: North-Holland, 1992

[12] T. H. Cormen, C. E. Leiserson, and R. H. Rivest, Introduction to Algorithms. Cambridge, MA: MIT Press, 1989.

[13] R. K. Ahuja, T. L. Magnanti, and J. B. Orlin, Network Flows: Theory, Algorithms, and Application. Englewood Cliffs, NJ: Prentice-Hall, 1993.

[14] D. F. Wong and C. L. Liu, "A new algorithm for floorplan design," in Proc. Design Automation Conf., 1986, pp. 101-107. 